\title{
Search for CP Violating neutral Higgs bosons in the MSSM at LEP
}

\section{Philip Bechtle ${ }^{* \dagger}$}

Stanford Linear Accelerator Center, 2575 Sand Hill Road, Menlo Park, CA 94025, USA

E-mail: bechtleeslac.stanford.edu

The LEP collaborations ALEPH, DELPHI, L3 and OPAL have searched for the neutral Higgs bosons which are predicted within the framework of the Minimal Supersymmetric Standard Model (MSSM). The data of the four collaborations are statistically combined and show no signicant excess of events which would indicate the production of Higgs bosons. The search results are thus used to set upper bounds on the cross sections of various Higgs-like event topologies and limits on MSSM benchmark models, including CP-conserving and CP-violating scenarios. Here, the limits on the model parameters of the CP-violating benchmark scenario CPX and derivates of this scenario are shown.

International Europhysics Conference on High Energy Physics

July 21st - 27th 2005

Lisboa, Portugal

\footnotetext{
* Speaker.

${ }^{\dagger}$ For the LEP collaborations
} 


\section{Introduction}

It is generally assumed that the Higgs mechanism [1] is responsible for the breaking of electroweak symmetry and for the generation of elementary particle masses, both in the Standard Model and in one of its most popular extensions, Supersymmetry (SUSY) [2]. In the implementation of SUSY with minimal additional particle content, the Minimal Supersymmetric Standard Model (MSSM), two Higgs doublets are needed. Hence, three neutral Higgs bosons and a pair of charged Higgs bosons can be searched for.

Most of the experimental investigations carried out in the past at LEP and elsewhere were interpreted in MSSM scenarios which assume CP conservation in the Higgs sector. However, CP violation in the Higgs sector cannot be a priori excluded [3]. Scenarios with CP violation are theoretically appealing since they provide one of the ingredients needed to explain the observed cosmic matter-antimatter asymmetry. In the MSSM, however, substantial CP-violating effects can be induced by radiative corrections, especially from third generation scalar-quarks. In the CPviolating scenario the three neutral Higgs mass eigenstates, $\mathrm{H} 1, \mathrm{H} 2$ and $\mathrm{H} 3$, are mixtures of $\mathrm{CP}$ even and CP-odd fields. The Higgs boson production and decay properties may therefore be widely different and, consequently, the experimental exclusions published so far for the CP-conserving MSSM scenario may be invalidated by CP-violating effects.

In this paper, the results from the combination of the Higgs boson searches of the four LEP collaborations [4] at $\sqrt{s}=91-209 \mathrm{GeV}$ in model-independent cross-section limits on various MSSM-Higgs-like topologies and in exclusion of CP-violating MSSM benchmark scenarios are presented.

\section{Higgs Boson Searches at LEP}

In the CP-conserving MSSM, the two dominant production mechanisms Higgsstrahlung $\left(\mathrm{e}^{+} \mathrm{e}^{-} \rightarrow \mathrm{hZ}, \sigma_{\mathrm{hZ}} \propto \sin ^{2}(\beta-\alpha)\right)$ and pair production $\left(\mathrm{e}^{+} \mathrm{e}^{-} \rightarrow \mathrm{hA}, \sigma_{\mathrm{hA}} \propto \cos ^{2}(\beta-\alpha)\right)$ are complementary and ensure the coverage of the whole kinematically accessible plane of Higgs boson masses, because for large $\cos ^{2}(\beta-\alpha)$ the two Higgs bosons $\mathrm{h}(\mathrm{CP}$-even) and $\mathrm{A}$ are close to each other in mass.

In the CP-violating MSSM, the experimental coverage of the mass plane is lost, since first all three Higgs bosons can be produced in Higgsstrahlung (and hence the direct complementary of two modes is lost), and second, because there can be large mass differences between $m_{\mathrm{H}_{1}}$ and $m_{\mathrm{H}_{2}}$ over the whole parameter space. Additionally, the cascade decay $\mathrm{H}_{2} \rightarrow \mathrm{H}_{1} \mathrm{H}_{1}$ is dominant in large areas of the parameter space. Hence, the coverage of non-diagonal pair production mechanisms and the coverage of cascade decays is crucial for the experimental access to the CP-violating models. This is shown in Fig. 1. In (a), the $95 \%$ confidence level (CL) exclusion limits on $\sigma \times B R$ in the process $\mathrm{e}^{+} \mathrm{e}^{-} \rightarrow \mathrm{H}_{2} \mathrm{Z} \rightarrow \mathrm{H}_{1} \mathrm{H}_{1} \mathrm{Z} \rightarrow \mathrm{b} \bar{b} b \bar{b} \mathrm{Z}$ relative to the nominal SM cross-section is shown. For $m_{\mathrm{H}_{2}}$ up to $105 \mathrm{GeV}$ and all $m_{\mathrm{H}_{1}}$, models which predict a $\sigma \times \mathrm{BR}$ value of more than $40 \%$ of the SM crosssection can be excluded. In (b), the coverage for the process $\mathrm{e}^{+} \mathrm{e}^{-} \rightarrow \mathrm{H}_{2} \mathrm{H}_{1} \rightarrow \mathrm{H}_{1} \mathrm{H}_{1} \mathrm{H}_{1} \rightarrow \mathrm{b} \bar{b} b \bar{b} b \bar{b}$ is shown, with limits relative to the nominal pair-production cross-section with $\cos ^{2}(\beta-\alpha)=1$. 

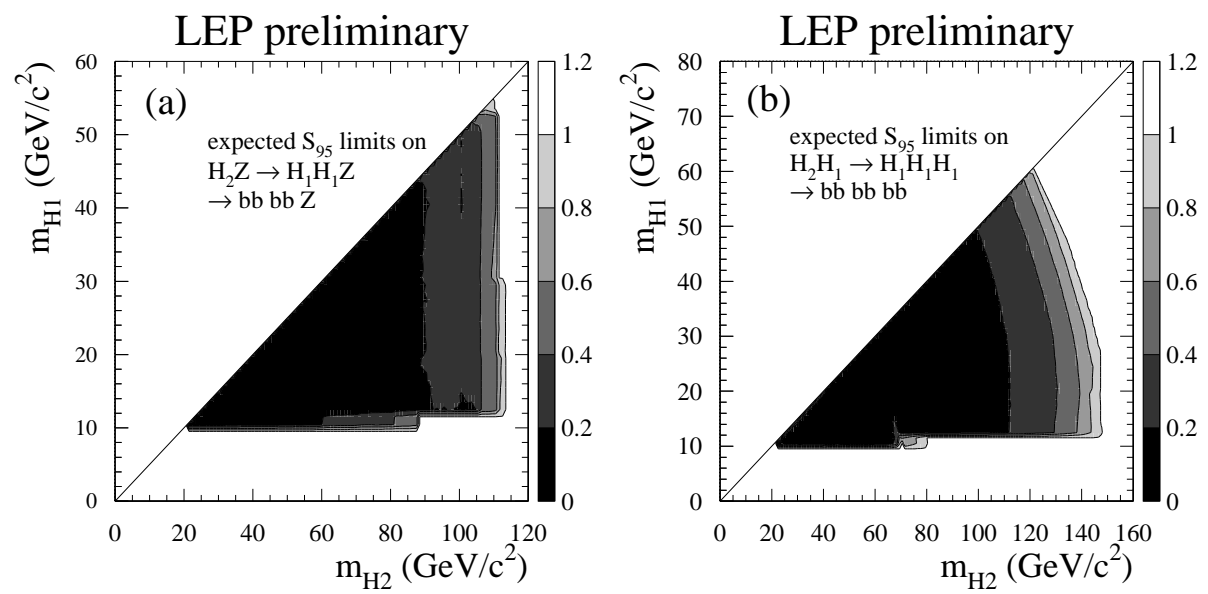

Figure 1: Model independent limits on $\sigma \times \mathrm{BR}$ relative to the nominal $2 H D M$ cross-sections for $\sin ^{2}(\beta-$ $\alpha)=1$ in (a) and $\cos ^{2}(\beta-\alpha)=1$ in (b).

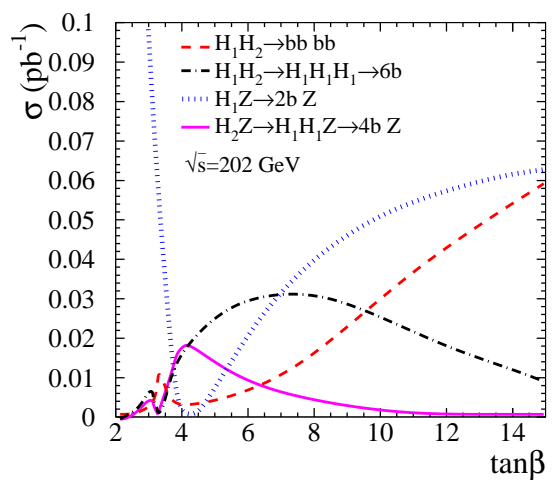

Figure 2: Model predictions for $\sigma \times \mathrm{BR}$ values of dominant Higgs boson production mechanisms in the $C P X$ scenario for $30 \mathrm{GeV}<m_{\mathrm{H}_{1}}<40 \mathrm{GeV}$.

\section{MSSM Models with Additional CP Violation}

The benchmark model used in the combination of the LEP data is the CPX scenario [5]. It is characterized by large mixing between CP-even and CP-odd states in the mass eigenstates. The CP-even/CP-odd mixing $\mathscr{M}_{S P}^{2}$ is characterized by

$$
\mathscr{M}_{S P}^{2} \propto \frac{m_{t}^{4}}{v^{2}} \frac{\mathfrak{I}\left(A_{t, b} \mu\right)}{M_{S U S Y}^{2}} .
$$

Therefore, large values of the top quark mass $m_{t}$, the Higgsino mixing parameter $\mu$ and the imaginary part of the trilinear couplings in the stop and sbottom sector $A_{t, b}$, coupled with a not too large scale of the squark masses $M_{S U S Y}$ is chosen. Effects of the variation of these parameters are studied. Detailed calculations on the two-loop order [6] or on the one-loop renormalization-improved order [5] are used to calculate the model predictions.

The resulting predictions for selected processes in the CPX scenario are shown in Fig. 2 for lightest Higgs masses of $30 \mathrm{GeV}<m_{\mathrm{H}_{1}}<40 \mathrm{GeV}$. For low $\tan \beta=v_{2} / v_{1}$, the SM-like production 

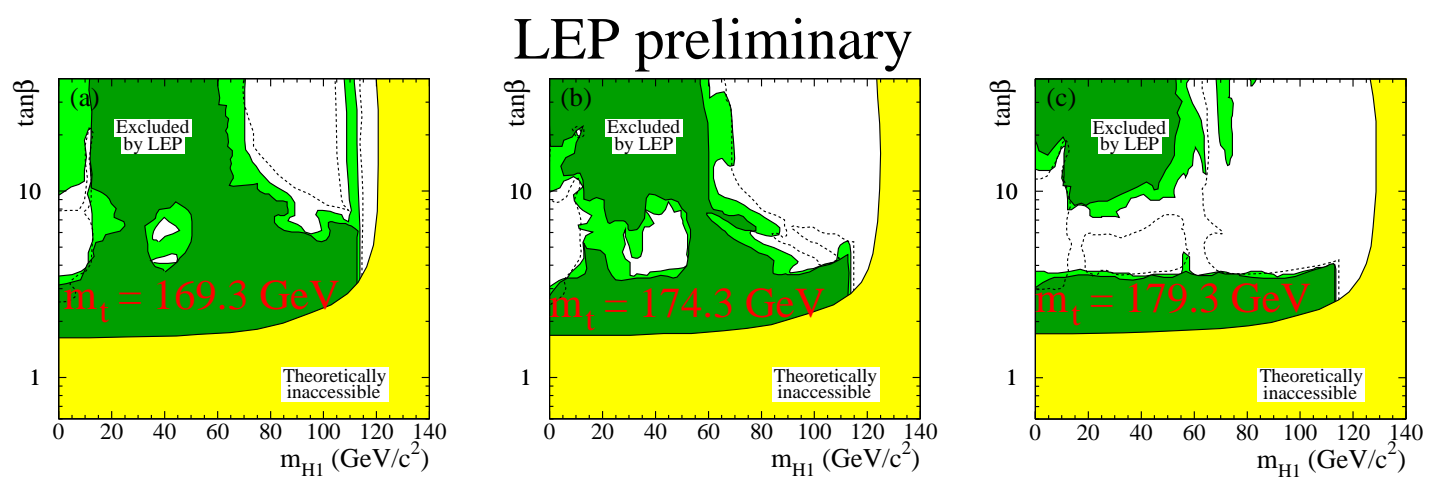

Figure 3: Exclusion areas in the $\left(\tan \beta, m_{\mathrm{H}_{1}}\right)$ plane in the CPX scenario for $m_{\mathrm{t}}=169.3,174.3,179.3 \mathrm{GeV}$. Theoretically inaccessible regions are shown in yellow, experimentally excluded areas in light green $(\mathrm{CL}=$ $95 \%)$ and dark green $(\mathrm{CL}=99.7 \%)$.

mechanism $\mathrm{H}_{1} \mathrm{Z} \rightarrow\left(\mathrm{b} \bar{b}, \tau^{+} \tau^{-}\right) \mathrm{Z}$ is dominant and has a large production cross-section. For intermediate $\tan \beta$, however, all production cross-sections are reduced, since the kinematically accessible $\mathrm{H}_{1}$ decouples from the $\mathrm{Z}$, hence no Higgsstrahlung occurs, and since $m_{\mathrm{H}_{2}} \approx 110 \mathrm{GeV}$ is close to the kinematic limit. Additionally, the experimentally more difficult cascade decay $\mathrm{H}_{2} \rightarrow \mathrm{H}_{1} \mathrm{H}_{1}$ becomes dominant. For large $\tan \beta$ the production cross-sections increase and finally $\mathrm{H}_{1} \mathrm{H}_{2} \rightarrow b \bar{b} b \bar{b}$ becomes the dominant mode.

\section{Interpretation of the LEP Data in the CPV MSSM}

The statistical combination of all Higgs boson searches from all four LEP collaborations uses the modified frequentist approach as implemented in [7]. The result of this combination shows no statistically significant excesses of the data over the expected background. Hence, limits on the parameter space are computed [8]. These limits are shown in Fig. 3 and 4 for the CPX scenario. In each case, the full set of MSSM parameters is fixed to the values chosen for the scenario (as given in $[5,8]$ ), apart from $\tan \beta$ and the charged Higgs boson mass $m_{\mathrm{H}^{ \pm}}$, which are scanned. The result is then shown in the $\tan \beta, m_{\mathrm{H}_{1}}$ projection.

In Fig. 3 the results in the CPX scenario are shown for different top quark masses $m_{t}$. The present experimental value of $m_{t}=172.7 \mathrm{GeV}$ [9] lies between the values used for Fig. 3 (a) and (b). In all cases, the reduction of production cross-sections for intermediate $\tan \beta$ described in Section 3 causes unexcluded regions for low values of the lightest Higgs boson mass $m_{\mathrm{H}_{1}}$. No absolute limit on $m_{\mathrm{H}_{1}}$ can be set. For larger values of $m_{t}$ this effect increases, since $m_{t}$ strongly influences the mixing of the mass eigenstates (see (3.1)) and increases the mass splitting between $m_{\mathrm{H}_{1}}$ and $m_{\mathrm{H}_{2}}$, hence further decreasing the production cross-sections for intermediate $\tan \beta$.

The effect of unexcluded regions in the parameter space for low $m_{\mathrm{H}_{1}}$ is clearly connected to the CP-violating imaginary phase of the trilinear couplings $A_{t, b}$. This is shown in Fig. 4. Only for large phases (and hence large mixings in (3.1)) the effect of large inaccessible regions is strong.

\section{Conclusions}

The results from neutral Higgs bosons searches in the context of the MSSM described in this 

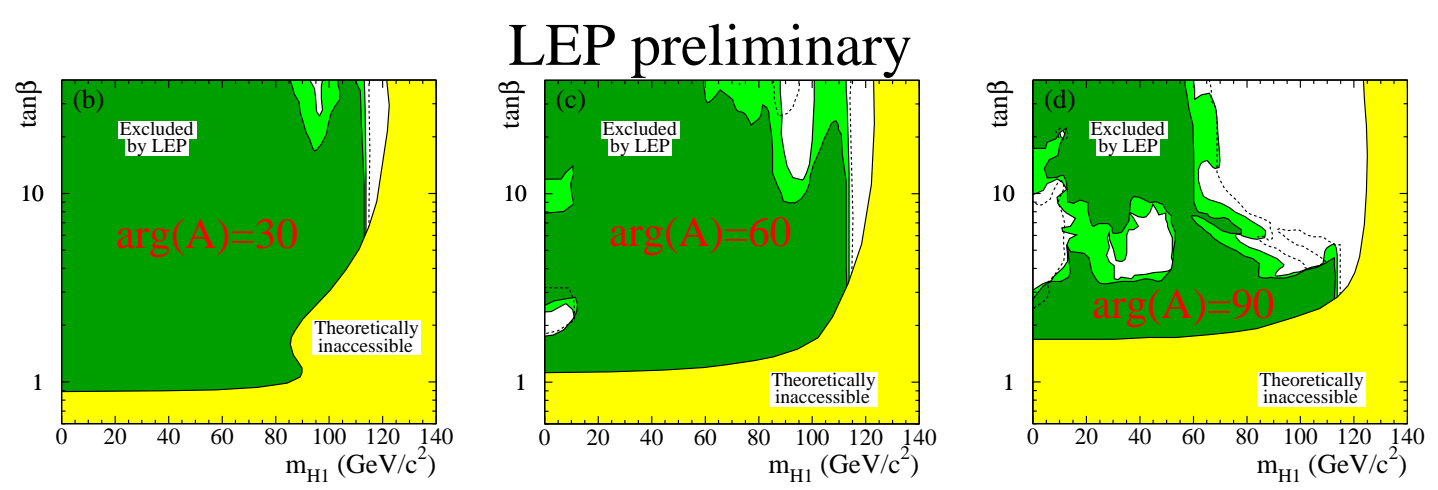

Figure 4: Exclusion areas in the $\left(\tan \beta, m_{\mathrm{H}_{1}}\right)$ plane for different values of the phase $\arg A$ of the trilinear coupling parameters in the stop and sbottom sector.

paper are based on data collected by the four LEP collaborations, ALEPH, DELPHI, L3 and OPAL at $\sqrt{s}=91-209 \mathrm{GeV}$. No significant excess of data over the expected backgrounds has been found. From these results, upper bounds are derived for the cross sections of a number of Higgs event topologies. These upper bounds cover a wide range of Higgs boson masses and are typically much lower than the largest cross sections predicted within the MSSM framework. In the CPviolating benchmark scenario CPX and the variants which have been studied, the combined LEP data show large unexcluded domains, down to the smallest masses; hence, no absolute limits can be set for the Higgs boson masses. On the other hand, $\tan \beta$ can be restricted to values larger than 2.9 for $m_{t}=174.3 \mathrm{GeV}$. While the excluded mass domains vary considerably with $m_{t}$, the bound in tan is barely sensitive to the precise choice of the top quark mass.

\section{References}

[1] P. W. Higgs, Phys. Rev. Lett. 13(1964) 508;

F. Englert and R. Brout, Phys. Rev. Lett. 13(1964) 321;

G. S. Guralnik, C. R. Hagen, and T. W. B. Kibble, Phys. Rev. Lett. 13(1964) 585.

[2] J. Wess and B. Zumino, Nucl. Phys. B70(1974) 39.

[3] A. Pilaftsis and C. E. M. Wagner, Nucl. Phys. B553(1999) 3.

[4] ALEPH Collab., A. Heister et al., Phys. Lett. B526 (2002) 191;

DELPHI Collab., J. Abdallah et al., Eur. Phys. J. C32 (2004) 145;

L3 Collab., P. Achard et al., Phys. Lett. B545 (2002) 30;

OPAL Collab., G. Abbiendi et al., Eur. Phys. J. C37 (2004) 49.

[5] M. Carena, J. R. Ellis, A. Pilaftsis and C. E. Wagner, Phys. Lett. B 495 (2000) 155.

[6] S. Heinemeyer, W. Hollik, and G. Weiglein, Comput. Phys. Commun. 124(2000) 76;

M. Frank, S. Heinemeyer, W. Hollik, and G. Weiglein, arXiv:hep-ph/0212037 (2002).

[7] T. Junk, Nucl. Instrum. Meth. A434(1999) 435;

P. Bock, arXiv:hep-ex/0405072.

[8] ALEPH, DELPHI, L3 and OPAL Collaborations, A. Heister et al., "Search for Neutral MSSM Higgs Bosons at LEP”, LHWG-Note 2005-01 (2005).

[9] CDF and D0 Collaborations, arXiv:hep-ex/0507091 (2005). 